\title{
Research Residuals Weaken Multibeam Bathymetric Data Based on Curve Fitting and Wavelet Transform
}

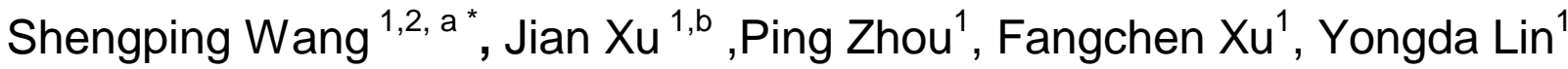 \\ ${ }^{1} 418$ Guanglan Road, Changbei Economic Development Zone ,Nanchang,Jiangxi,China \\ ${ }^{2}$ Key Laboratory of Watershed Ecology and Geographical Environment Monitoring,NASMG, \\ Nanchang,330013,China \\ a shpwang@ecit.cn, ${ }^{b} x u j i a n x j 92 @ 163 . c o m$
}

Keywords: multi beam sounding; residual error; least square curve fitting; wavelet transform Abstract: the residual error of the left and right ping data in the multi beam sounding is obvious, and it is not easy to be extracted. In this paper, we use the least square curve fitting and wavelet transform to analyze the residual error, verify it's feasibility by the method of doing experiments.

\section{Introduction}

Ocean survey is conducted under the condition of sea water movement. Multi beam swath bathymeter is composed of multi sensor integrated sounding system, except by its own measurement error, but also affected by the velocity, attitude, draft, with stitching error of angle and installation, and a band edge error (showing the "bulge" or "sag" was the main source of residual error. In this paper, we study the principle of least squares curve fitting and wavelet transform theory, and weaken the residual error, and get the real seabed terrain data.

\section{Curve fitting and wavelet transform theory}

curve fitting theory. According to curve fitting theory, we conduct center beam to curve fitting and weaken "heave" and "hollow" of edge beam to approach real topography of Ping section, and then finding the optimal appraisement according to least squares method.

Normally, on the basis of complexity of real topography in ping section undersea we select the order of polynomial trend curve, general type of polynomial curve function is:

$$
f(x)=a_{0}+a_{1} x+a_{2} x^{2}+a_{3} x^{3}+\mathrm{L}+a_{n} x^{n}=\sum_{i=0}^{n} a_{n} x^{n}
$$

In formula (2.1.1), $a_{i}(i=1,2, \mathrm{~L} n)$ is coefficient of polynomial curve, it is also unknown parameter, $P_{n}\left(x_{i}, z_{i}\right)$ is coordinate of ping section.

The total deviation square is :

$$
S S T=\sum_{i=0}^{n}\left[\left(x-y x_{0}^{2}+\left(Z-2 x_{0}^{2}\right]=\min \right.\right.
$$

wavelet transform theory. Multiple-beam sounding data is belong to unstable data. Therefore, we use wavelet transform derived by Fourier transform to process data, then accurate positioning high frequency signal and collecting or removing it.

Dispersing parameters $\mathrm{a}$ and $\mathrm{b}$ in function $\psi_{a, b(x)}$ in wavelet function, making $a=a_{0}^{p}, b=a_{0}^{p} q$, then we can get wavelet function:

$$
y_{p, q(x)}=a_{0}^{-p / 2} y\left(a_{0}^{-q / 2} x-q\right)
$$

If $f\left(N_{\Delta x}\right),(\Delta x$ is data sampling interval, $N \in 1,2,3 \mathrm{~K} n$ ), we can get $f(x)$ discrete wavelet transform: 


$$
(W f)(p, q)=a_{0}^{-p / 2} \int_{m}^{n} f(x) \bar{y}\left(a_{0}^{-j} x-q\right) d t
$$

Residual error data is changed gently, we choose gentle wavelet function processing, after experimental analysis we use Db4 wavelet function:

Making $H(\varphi)$ function as :

$$
H(a)=\left[\frac{1+e^{-i a}}{2}\right]^{N} L\left(e^{-i a}\right)=\frac{1}{\sqrt{2}} \sum_{k=0}^{2 N-1} h_{k} e^{-i a k}
$$

Establishing double scale equation to push out Db4 wavelet function:

$$
\psi(x)=\sqrt{2} \sum_{n}(-1)^{1-n} \bar{h}_{1-n} \phi(2 x-n)
$$

Taking formula (2.2.4) into formula (2.2.2) we can get wavelet transform model, and using this method to scale up signal of edge strip data, setting threshold value can automatic withdrawal usable data, adopting:

$$
\begin{gathered}
\Delta Z=Z-Z_{0} \\
Z^{\prime}=\Delta Z+Z_{0}^{\prime}
\end{gathered}
$$

Adding the signal into curve fitting data, it can approach real submarine topography accurately, weakening residual error in edge strip more.

\section{Experimental analysis of curve fitting and wavelet transform}

Research method process:

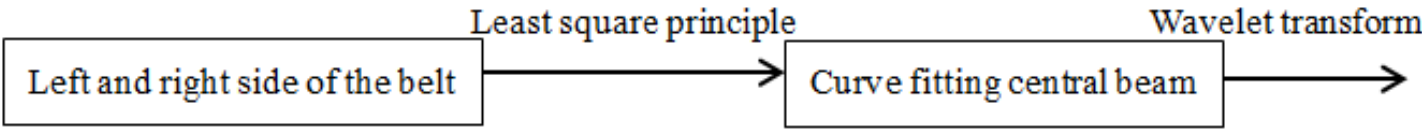

Elimination of residual error

Fig. 1 Research method process

Experimental analysis of least square curve fitting. In sea water, the sound velocity, attitude, draft, installation error, temperature, salinity, static pressure and the edge of the residual error caused by the residual error, resulting in the existence of the data in the presence of the edge of the adjacent band of the regular "uplift" or "depression"". In this paper, we study "uplift" as an example. As shown in Figure 2:

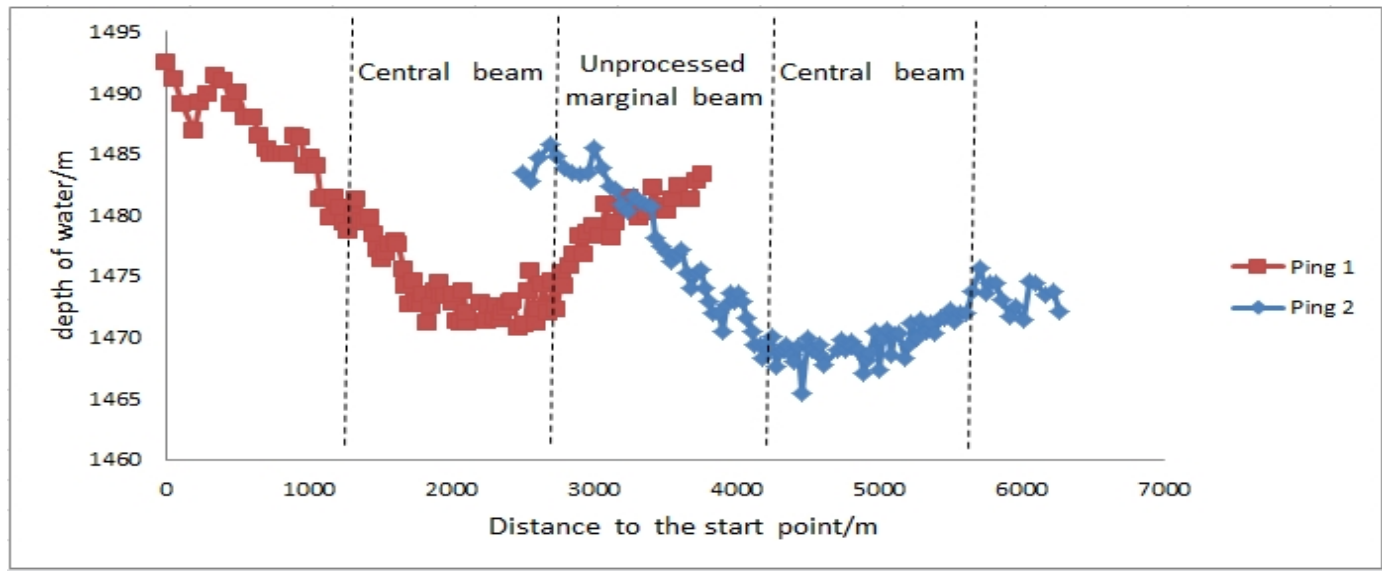

Fig. 2 Raw data 
Compared with marginal beam, the data error of central beam is small, and the fitting curve of the two central beam is obtained by using the least square principle. The experimental order of this experiment is 2, 3 and 4. As shown in Figure 3:

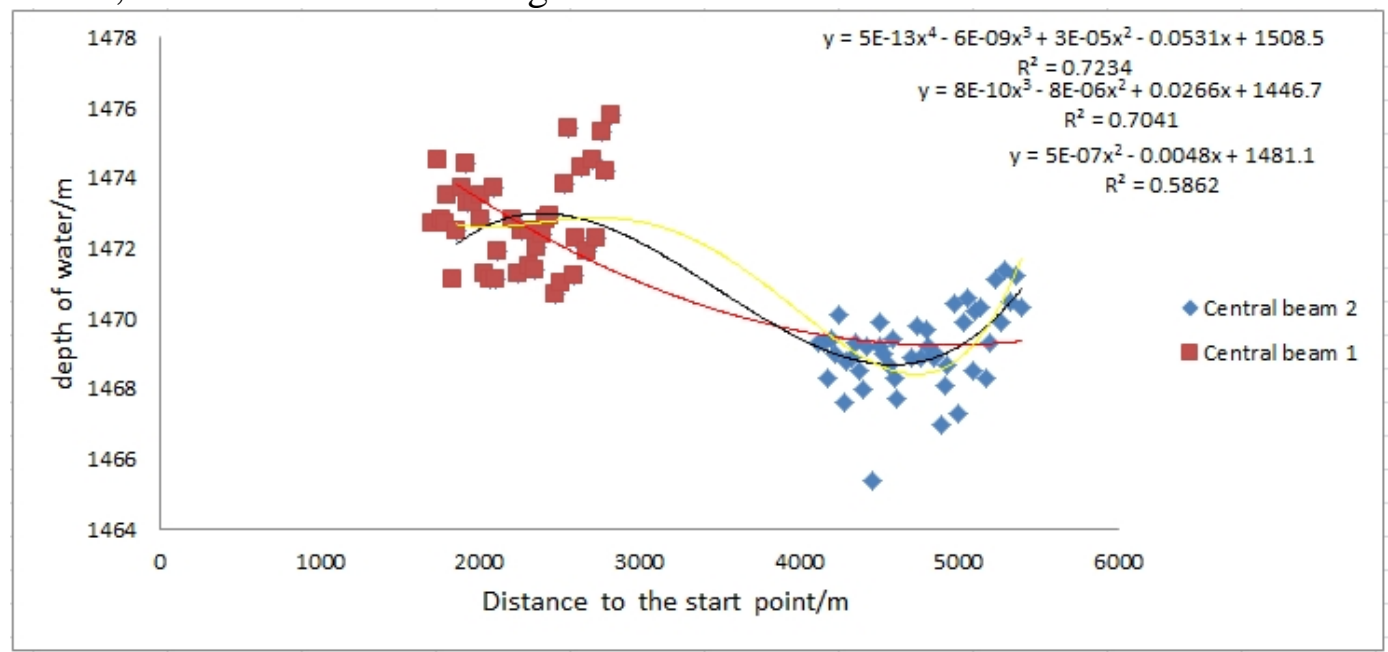

Fig. 3 Central beam curve fitting

In Figure 3, the fitting number of red (lower), black (middle) and yellow (up) curves is 2, 4 and 3 times, and the curve change trend is obvious. That 3 times fitting curve can effectively weakening edge banding of residual error, and good retention of the original marine data information. So, for the same region, the selection of the order is particularly important, the experimental selection of the order of the 3 fitting curve.

\section{Wavelet transform to extract weak signals experimental analysis}

The selection of wavelet function is the key to extract the high frequency signal in the process of extracting the edge beam. Therefore, the MATLAB, Db4 and Sym4 wavelet functions are used to analyze the data of the edge strip, and the experimental results are compared and analyzed.As shown in table 1:

Table 1:Comparative analysis of statistical parameters of wavelet function

\begin{tabular}{lllllc}
$\begin{array}{l}\text { Statistical } \\
\text { parameters }\end{array}$ & Mean & Median & $\begin{array}{l}\text { Standard } \\
\text { Deviation }\end{array}$ & $\begin{array}{l}\text { Median } \\
\text { Deviation }\end{array}$ & $\begin{array}{c}\text { Mean } \\
\text { Deviation }\end{array}$ \\
\hline Bior3.9 & -0.00114 & -0.3836 & 2.54 & 1.745 & 2.076 \\
Db4 & 0.001057 & -0.3283 & 2.226 & 1.602 & 1.863 \\
Sym4 & -0.00849 & -0.3264 & 2.615 & 1.798 & 2.149 \\
\hline
\end{tabular}

The statistical parameters of Bior3.9, Db4 and Sym4 show that the statistical parameters of Db4 wavelet function are smaller than those of the other two wavelet functions. Therefore, the Db4 wavelet function is the most accurate and effective, and then the high frequency signal is added to the fitting curve.

\section{Combined curve fitting and wavelet transform data processing analysis}

After curve fitting and wavelet transform data processing before and after comparison, the effect is shown in Figure 4: 


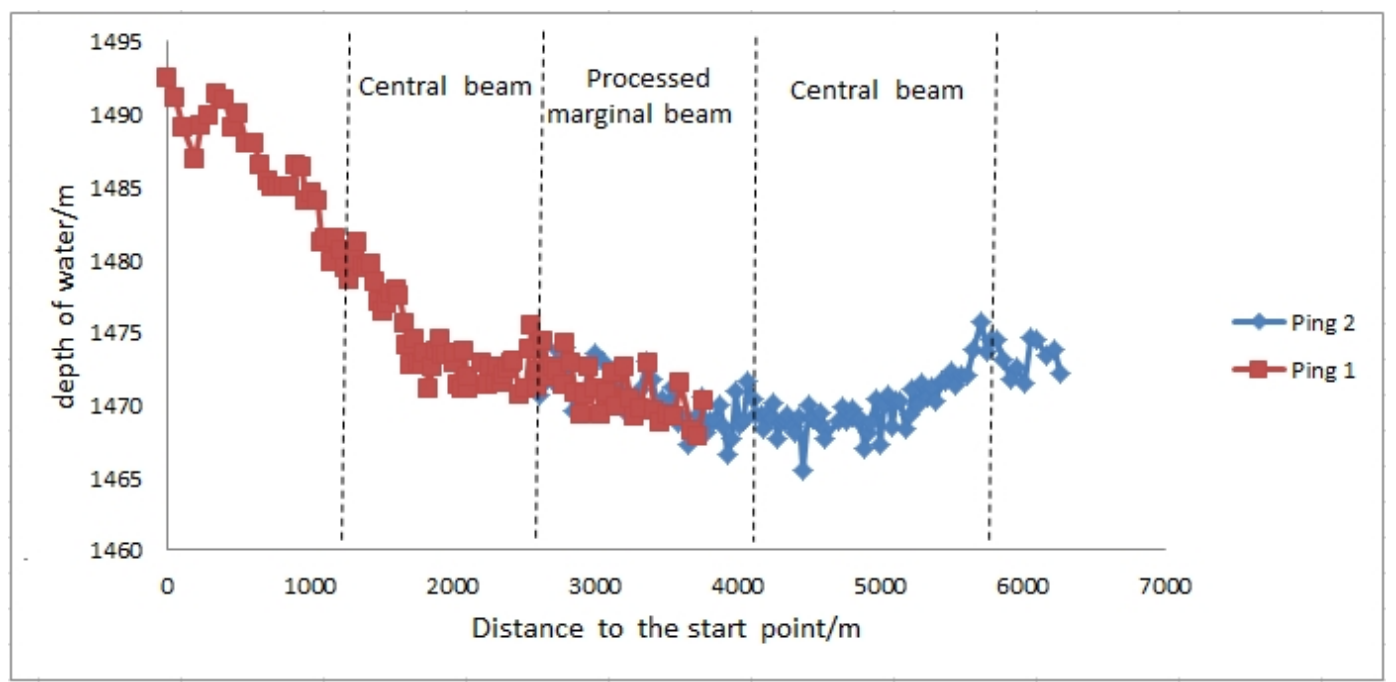

Fig. 4 Processed marginal beam

Through figure 4, it can be seen that after data processing, the two parts of the "raised" with a marked weakening, the edge of the beam is no longer a big "bulge", a relatively flat, two with a close agreement with the coverage. The results of this study are consistent with the real terrain of the sea floor, and the conclusion is drawn based on curve fitting and wavelet transform to weaken the feasibility of multi - beam sounding data.

\section{Assessment of effect}

Based on above methods, we use principle of least squares to realize curve fitting of center beam, then use wavelet transform to extract high frequency signal in public edge strip beam and add the signal to fitting curve to weaken sounding residual error in both left and right sides, finally we conduct experimental analysis and accuracy evaluation and verify the superiority of this method.

Curve fitting evaluation of center beam. Comparing with the maximum, the minimum of before and after manipulation, coefficient of determination and variance, we can assess effect of curve fitting. If variance MSE is equal to 0 (or very close to 0 ) and the more coefficient of determination in close to $1(\mathrm{R}$ - square $\leq 1)$, the better effect of center beam data curve fitting.

Center beam data is collection $P_{n}\left(x_{i}, y_{i}\right)$, the corresponding center beam data point collection is $B_{n}\left(\tilde{x}_{i,} \tilde{y}_{i}\right)$, and it will be:

$$
M S E=\frac{\sum_{i=0}^{n}\left[\left(x_{i}-\ell_{i}\right)^{2}+\left(y_{i}-y_{i} /\right)^{2}\right]}{n}
$$

Assessing R-square ( coefficient of determination ) :

$$
R-\text { square }=1-\frac{n * M S E}{S S T}
$$

Table 2 curve fitting evaluation of center beam

\begin{tabular}{llc}
\hline $\begin{array}{l}\text { Statistical } \\
\text { parameter }\end{array}$ & MSE & R-square \\
\hline $\begin{array}{l}\text { Before } \\
\text { manipulation }\end{array}$ & 17.06817 & 0.855 \\
$\begin{array}{l}\text { After } \\
\text { manipulation }\end{array}$ & 16.20064 & 0.891 \\
\hline
\end{tabular}


From table 2 we can see,that the effect of center beam curve fitting is good, coefficient of determination R-square is close to 1 , curve correlation is high, but fitting effect shown by variance MSE is not obvious, data accuracy is low.

data evaluation after wavelet transform. Using wavelet transform to obtain high frequency signal and process evaluation of residual error weakening effect, we can conduct evaluation of consistency in overlapping region in virtue of adjacent strip beam sounding data. There exists a pair of sounding data parameters to evaluate in left and right beams in overlapping region.

Table 3 consistency evaluation parameters of adjacent strip sounding data in overlapping region before and after wavelet transform

\begin{tabular}{lcccc}
\hline Statistical parameter & $\begin{array}{l}\text { Maximum } \\
\text { deviation/m }\end{array}$ & $\begin{array}{l}\text { Minnimum } \\
\text { deviation/m }\end{array}$ & $\begin{array}{l}\text { standard } \\
\text { deviation/m }\end{array}$ & $\begin{array}{l}\text { R-square after } \\
\text { transformation }\end{array}$ \\
\hline $\begin{array}{l}\text { Before wavelet } \\
\text { transfoem }\end{array}$ & 6.5 & 0.1 & \pm 2.432 & 0.891 \\
$\begin{array}{l}\text { After wavelet } \\
\text { transform }\end{array}$ & 1.9 & 0.1 & \pm 0.678 & 0.941 \\
\hline
\end{tabular}

From table 3 we can know, the maximum, the minimum and standard deviation are obviously reduced, standard deviation is close to 0 , R-square (coefficient of determination) is close to 1 , it fully proves after correction the weakening effect of data is obviously enhanced. After evaluation of manipulation we can conclude that wavelet transform can effectively weaken edge beam residual error.

\section{Conclusion}

Comparing before with after manipulation of edge beam data in virtue of least squares curve fitting and wavelet transform method, apply of wavelet transform solves the difficulty of extracting weak signal in residual error, multiple-beam sounding data after manipulation is match with theory effect of data image, it is approach to real submarine topography.

In addition, in other manipulation methods, such as similar triangles proportion method, we needs aplenty of experiments to confirm the optimal proportionality coefficient, and the process it complex. The method based on curve fitting and wavelet transform to weaken multiple-beam sounding residual error in this article is convenient of programming realization, effect is remarkable. Experiment research shows that using textual research method to manipulate data is closest to real submarine topography, it concludes that this method is feasible in multiple-beam data processing.

Supported by: National Natural Science Foundation of China(NO.: 41206078);Natural Science Foundation of Jiangxi Province(NO.:20142BAB217025);Humanities and Social Sciences Foundation of Jiangxi Province(NO.: GJJ13441); Transportation hall of science and technology plan projects of Guangdong province(NO.: Science and technology -2013-02-048).

\section{References}

[1] Yu Jiacheng, Xu Xueqiang .Digital Bathymetry Model for Multibeam Based on Attitude and Terrain Effect[J]. Journal of System Simulation,2015,04:824-829.

[2] Lu Dong, Chen Baowei, Li Haisen, Zhou Tian. Technology of the multi-beam high frame rate bathymetry in shallow water[J]. Journal of Applied Acoustics,2015,04:303-310.

[3] Huang Chenhu,Lu Xiuping,OuYang Yongzhong,Ning Linchun,Huang Xianyuan,Deng Zhiju. Analysis of Error Source and Quality Assessment Multibeam Sounding Product[J]. Hydrographic Surveying and Charting,2014,02:1-6.

[4] Zhao Jianhu,Zhang Hongmei, Yang Jun. Weaking Influence of Residual Error of MBS Sounding[J]. Geomatics and Information Science of Wuhan University. 2013,10:1184-1187.

[5] Chen Liangbo,Zheng Yaqing.Study on Curve Fitting Based on Least Square Method[J].Journal of Wuxi Institute of Technonlogy ,2012,05:52-55. 
[6] Li Jian,Zhou Yunxuan,Xu Huiping.The Selection of Wavelet Generation Functions in Data Processing of Gravity Field.Geophysical and Geochemical Exploration[J]. 2001,06:410-417. 